\title{
CLARO S.A. Y EL DESARROLLO DE LAS TELECOMUNICACIONES EN COLOMBIA
}

\author{
Diana Patricia Castañeda Garzón \\ Facultad de Negocios, Gestión y Sostenibilidad \\ Programa de Especialización en Gestión empresarial \\ dianacastaneda82@hotmail.com \\ Ricardo Pachón Mantilla \\ Facultad de Negocios, Gestión y Sostenibilidad \\ Programa de Especialización en Gestión empresarial \\ ricardo.pachon@sandvik.com \\ Sulied Fernanda Salazar Díaz \\ Facultad de Negocios, Gestión y Sostenibilidad \\ Programa de Especialización en Gestión empresarial \\ sulisaldi22@gmail.com
}

\section{Resumen}

El presente artículo muestra cómo ha sido el desarrollo de Claro S.A. y como sus estrategias han influenciado el sector de las telecomunicaciones en Colombia, las regulaciones iniciales a las que fue sometido el sector y como poco a poco se ha ido consolidando como uno de los sectores económicos más importantes del país.

Se realiza una prospectiva en general del negocio de las telecomunicaciones a nivel mundial y en particular en Colombia y como estas tendencias afectarían las decisiones a futuro de la empresa Claro y cuáles serían los retos a los cuales se enfrentaría.

Para la realización del presente artículo en forma de estudio de caso se utilizó una metodología cualitativa con alcance descriptiva, basada en el rastreo bibliográfico, encontrando que Claro Colombia es reconocida por ser una de las empresas más importantes y solidas del país, no solo por la prestación de sus servicios, ayudando a que los rincones más apartados del país se encuentren comunicados con las principales ciudades, sino que también es considerada como una de las empresas generadoras de empleo predilecta por los colombianos.

Además, Claro Colombia desde sus inicios ha sentado las bases de la transformación tecnológica del negocio de telecomunicaciones en el país, se ha caracterizado por estar a la vanguardia en prestación de servicios, infraestructura de telefonía móvil y fija, convirtiéndola 
en un elemento accesible a toda la población colombiana, ratificándose como la principal empresa de telecomunicaciones por su amplia cobertura.

Palabras clave: Estrategias, telecomunicaciones, telefonía móvil, cobertura, ingreso, mercado. 


\section{Introducción}

El presente artículo tiene como principal objetivo determinar si la llegada de la empresa Claro a Colombia, y su gestión para ser unas de las empresas de telecomunicaciones más grandes del país puede ser considerado como un caso empresarial de éxito.

Como objetivo secundario se define, conocer como el plan de desarrollo de una empresa y sus estrategias comerciales podrían contribuir al crecimiento de un país o región para un sector específico, así como estas estrategias pueden generar espacio para la creación de nuevas leyes que regulen o enmarquen todo un proceso dentro de un marco legal gubernamental. Así mismo, también se tiene como propósito realizar un análisis de la situación actual de Claro y cuáles serán sus principales desafíos a futuro.

Por último, se realizará un análisis de cómo ha manejado Claro la responsabilidad social empresarial desde su llegada al país y como la inclusión de la tecnología ha ido transformando los hábitos de la sociedad y generando cambios en costumbres anteriormente arraigadas.

En primera medida se resalta el desarrollo de las telecomunicaciones en Colombia y el papel que la empresa Claro ha jugado en el crecimiento del sector. Para tal fin, se dará comienzo con un breve recuento de la llegada de las telecomunicaciones al país, como han evolucionado a través de los años hasta llegar a la tecnología y masividad que presenta actualmente.

\section{Telecomunicaciones en Colombia}

Las telecomunicaciones actualmente están dentro de uno de los sectores económicos más grandes en el mundo, ya que son una parte fundamental en el progreso de la sociedad en general. La gran importancia y necesidad de la comunicación humana es lo que ha permitido que la tecnología cada vez más avanzada dedique parte de sus progresos a mejorar y facilitar los medios y modos de comunicación entre empresas, bancos, personas, en fin, entre todos los entes que conforman una sociedad (Benavides \& Castro, 2012). Por tal razón las empresas dedicadas al sector de las telecomunicaciones presentan un desafío a diario para estar a la vanguardia de estos avances tecnológicos y a la gran demanda social, permitiéndoles ser empresas competitivas y sostenibles en el tiempo. Por tal razón, se quiere estudiar si la llegada de la empresa Claro a Colombia ha aportado cambios significativos en la mejora de la comunicación en el país y si derivado de la gestión se ha permitido convertirse en una de las empresas de telecomunicaciones más grande del país.

\section{Antecedentes}

La historia de las telecomunicaciones en Colombia se remonta al año de 1847 donde se realiza el primer hito en telecomunicaciones en el país, "el telégrafo eléctrico"; desde esta época hasta el año 1913 no hubo grandes avances en el sector, fue en este año que la empresa Marconi Wireless inicio la prestación del servicio de telegramas en el país donde conformó 
una red extensa en 12 ciudades del país, en 1919 el gobierno contrató esta empresa para empezar con la instalación y montaje de la red internacional, la cual fue inaugurada cuatro años después y conectó a Colombia con el mundo; la empresa fue tan reconocida a nivel nacional que el telegrama adoptó coloquialmente el nombre de "Marconi".

En 1927 se decretó la construcción del palacio de las comunicaciones, el cual fue muy criticado debido a sus altos costos de construcción, finalmente este fue inaugurado en 1944 y podría ser considerado como la primera institución colombiana dedicada exclusivamente a fomentar y gestionar el sector de telecomunicaciones en el país.

En el año de 1.953 se crea oficialmente el Ministerio de comunicaciones estableciendo su funcionamiento con base en los departamentos de correos, telecomunicaciones y giros. (Acosta F, 2017)

Hasta la década de 1990 la telefonía estaba a cargo completamente del estado a través de diversas empresas municipales y de la empresa nacional Telecom. Con la Ley 37 de 1993, el gobierno nacional introdujo y reglamentó la telefonía móvil celular (TMC), permitiendo la entrada de operadores privados al negocio (CRT, 2002). En 1994 comienzan a funcionar las redes de telefonía celular a través de seis empresas fraccionadas en tres zonas de cobertura.

En cada zona operaba una empresa privada y una empresa mixta (capital privado y público, con la cooperación de las empresas de telefonía fija). Súbitamente estas empresas comenzaron a asociarse con el ingreso de capital privado para formar dos empresas de cobertura nacional: Comcel (controlada por América Móvil) y Telefónica (bajo su marca Movistar).

La desregulación de las telecomunicaciones facultó, que empresas locales como la Empresa de Telecomunicaciones de Bogotá (ETB) y Empresas Públicas de Medellín (E.P.M.) pudieran ofrecer servicios de larga distancia nacional e internacional a través de sus marcas 007 Mundo y Orbitel, así como Telecom y E.P.M entraron a prestar el servicio de telefonía local en la ciudad de Bogotá (bajo las empresas Capitel y EPM Bogotá).

En el año 2009 y debido principalmente a la masificación de la tecnología celular, el gobierno transformo el ministerio de comunicaciones en el ministerio de las TIC "Ministerio de la tecnología de la información y las comunicaciones", cuya función principal era adecuarse y establecer las regulaciones que garantizaran la libre competencia, el uso eficiente de la infraestructura y la protección de los derechos de los usuarios. (Gonzales, Sepulveda, \& Triviño, 2014)

Es así, que la historia de las telecomunicaciones en Colombia tiene varios hitos como la masificación del telegrama gracias a Marconi, el uso incremental del teléfono fijo en las residencias alrededor de los años 70, sin embargo el mayor hito en la historia reciente fue dado en el periodo entre los años 2000 - 2002, donde la penetración de la telefonía móvil fue superior a la telefonía fija y obligó al gobierno nacional a generar acciones de choque para entrar a regular inmediatamente este segmento del sector el cual hasta el momento carecía de 
un marco legal que pudiera garantizar el crecimiento adecuado manteniendo los derechos del consumidor como prioridad.

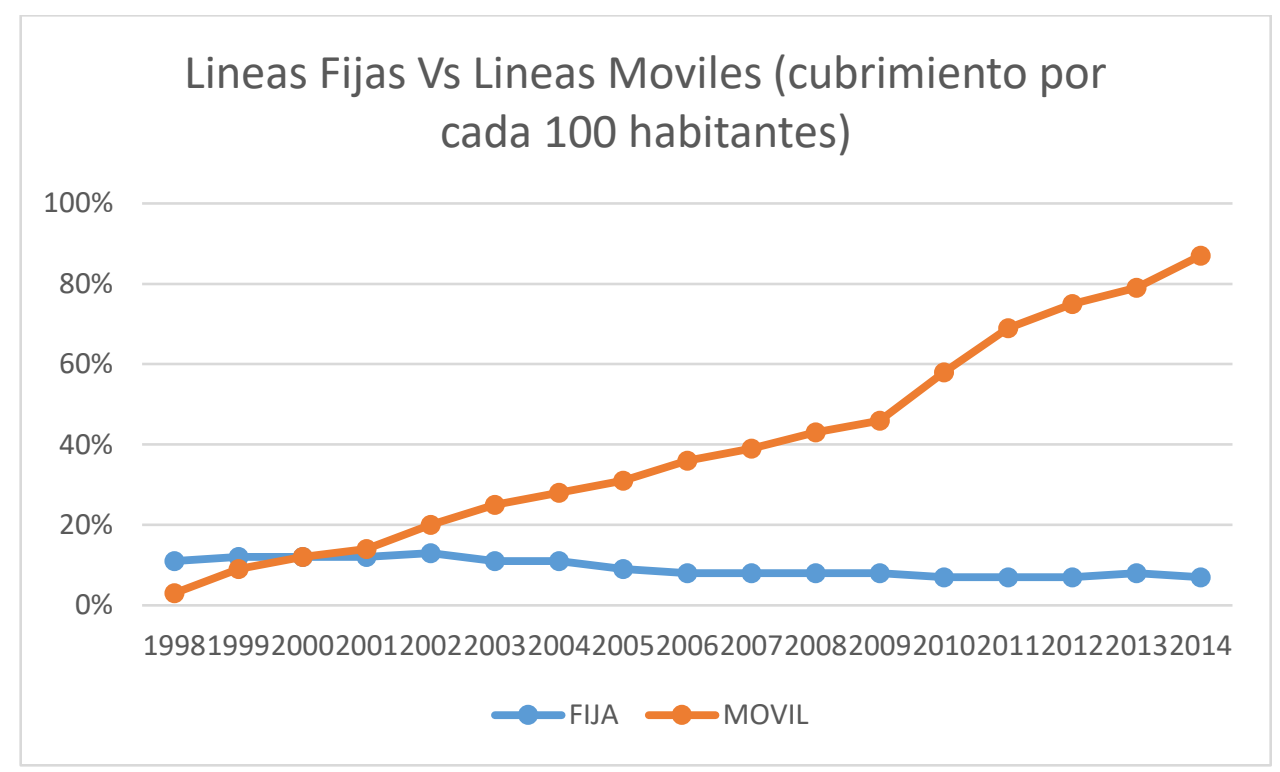

Gráficol. Indicadores TIC por cada 100 habitantes.

Fuente datos: Society Report 2016 UIT

Elaboración CRC

Sin lugar a dudas la evolución de la tecnología de las telecomunicaciones se encuentra a la vanguardia de los mayores avances tecnológicos de los últimos tiempos a nivel mundial, este ámbito abarca a todas las estructuras de la sociedad, de los negocios, los estados y las empresas, sin lugar a equivocarnos la tecnología de la información y las telecomunicaciones guiarán el desarrollo futuro del mundo, por ende es el sector donde se presentarán los más grandes desafíos ya que presentan ciclos de cambio rápido que son afectadas enormemente por las tendencias de la sociedad. (Herrera, 2008).

\section{Marco teórico}

\section{Ingreso multinacionales al mercado local de las telecomunicaciones.}

La ley 72 de 1989 eliminó la hegemonía estatal en el sector de las telecomunicaciones y con esta se abrió la puerta a que cualquier concesión privada pudiera prestar cualquier servicio en el sector. Un año después, la ley 1900 reguló todo lo necesario para la planeación y control tanto de los operadores como de los usuarios. (Congreso de la Republica, 2009) 
Las primeras empresas Extranjeras en ingresar al mercado colombiano fue Telefónica de España la cual adquirió el 52\% de la estatal Telecom por un valor de US\$360 millones de dólares; de esta adquisición nació Telefónica Colombia; esta compartiría el proceso de telefonía fija con el estado, sin embargo, tendría plenos derechos sobre la naciente telefonía móvil y sobre el sistema de televisión por suscripción. Finalmente, Telecom fue liquidado en el año 2003 y el gobierno colombiano dejó de tener cualquier participación en el negocio de las telecomunicaciones, pasando enteramente a manos privadas y el rol del gobierno fue solamente regulatorio.

En 1989 fue creada Celular S.A. que posteriormente fue llamada comunicación celular, Comcel S.A. la cual se instituyó como una empresa de capital mixto donde la empresa de teléfonos de Bogotá tendría el 57,85\% y Telecom con 21.36\%. En una segunda fase ingresó a la sociedad BCE Telecom INC proveniente de Canadá; la cual adquirió el 50\% de la empresa. (Sarmiento, 2011)

El gobierno debido al ingreso de empresas mixtas, un esquema nuevo de negocios en ese momento se vio obligado a generar un marco regulatorio donde dividió el país en tres zonas de prestación de servicios y reguló el espectro electromagnético donde asigno una franja a empresas privadas y otra a empresas mixtas.

Oficialmente Comcel S.A. inicio operaciones el 1 Julio de 1994; para 1997 Comcel lanza la "tarjeta amigo" que se convirtió en el primer servicio de uso de líneas móviles en modalidad de prepago; debido a este avance en la prestación del servicio y de adquisiciones estratégicas en infraestructura en este mismo año, Comcel se convierte en el primer operador en utilizar la red satelital para conectar a ciertas partes del territorio nacional donde no había hasta el momento cobertura de línea móvil; en este año Comcel empezó a significar entre los colombianos sinónimo de "cobertura".

Para 1999 Comcel seguía siendo el segundo operador más grande detrás de Bellsouth (antigua Telefónica), aunque ya se acercaba exponencialmente a su competidor; en el año 2001 Bell Canadá se asoció con la mexicana América Móvil, propiedad de Carlos Slim, y con southwestern Bell de Estados Unidos y se convertían en dueñas del 77.92\% de Comcel.

Para el 2002 Comcel cambia su estrategia y empieza a ampliar su infraestructura y pasaron de tener 270 a 350 estaciones logrando un cubrimiento del $96 \%$ del territorio nacional; debido a la liquidación de Telecom en 2003; Comcel compra esta participación y se hace al control del $100 \%$ de la empresa.

Como se pudo observar anteriormente las líneas móviles superaron a las líneas fijas entre los años 2.000 al 2.002, estos años coinciden con el plan de expansión de Comcel, su estrategia de inversión en infraestructura, la ampliación de la red satelital y la masificación del uso de las líneas móviles a través de la modalidad de prepago generaron los pilares del negocio de las telecomunicaciones tal con lo conocemos en la actualidad. (Torres, 2007). 
Para el año 2012, Comcel ya era de lejos el principal operador de telefonía móvil con más de 30 millones de usuarios; con una participación en el mercado de alrededor del $67 \%$ del mercado nacional; en este mismo año nace Claro Colombia S.A que resulta de la fusión de Comcel y Telmex, ambas, propiedad de Carlos Slim y de esta manera se fusionan los negocios de telefonía fija, telefonía móvil y televisión por suscripción.

\section{Claro en Colombia}

Claro Colombia S.A. inicia operaciones en junio de 2012; en este momento ya fungía como el líder del mercado; manteniendo hasta la actualidad su posición dominante. Sus políticas y estrategias han definido las directrices del mercado de las telecomunicaciones en los sectores de telefonía fija, telefonía móvil y televisión por suscripción; siendo las líneas móviles el reflejo de su participación dominante en el mercado.

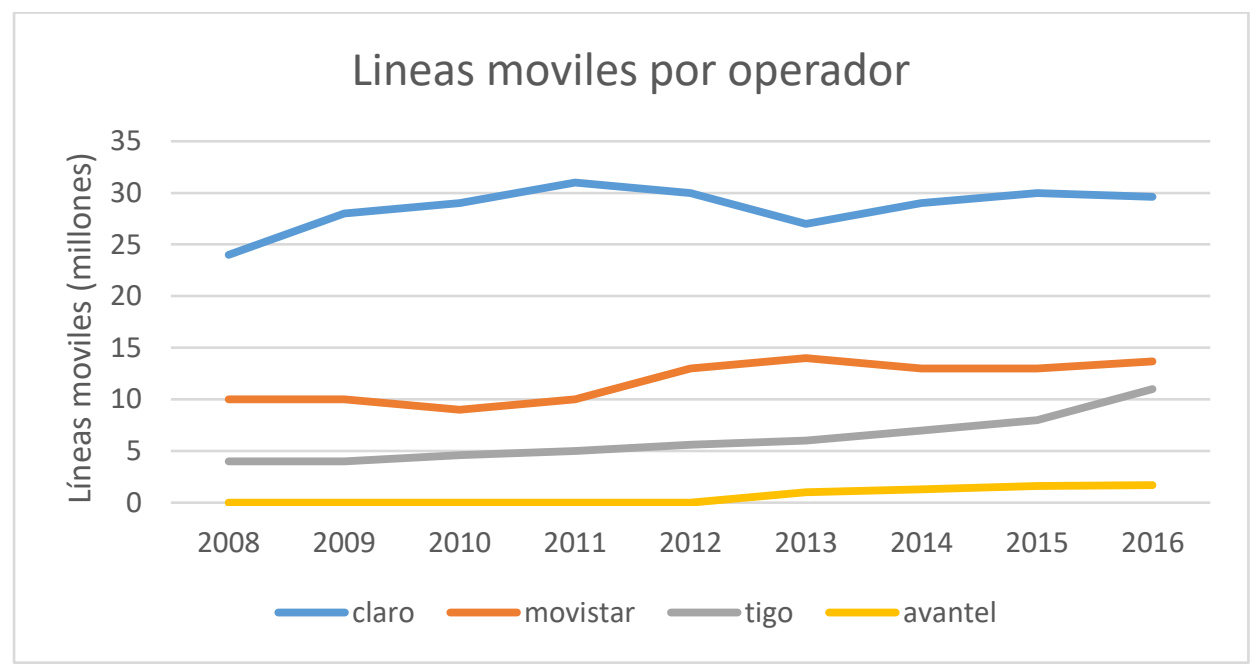

Gráfico 2. Líneas móviles por operador

Fuente: Datos del Mintic

Elaboración: Propia

En cuanto a televisión por suscripción, aunque se presenta un leve aumento de suscriptores en cifras comparadas para 2017, según datos de la Autoridad Nacional de Televisión (ANTV); en los últimos años se ha presentado un crecimiento exponencial de las plataformas digitales de televisión como Netflix; la cual aspira un nivel de suscriptores de 5 millones de usuarios; casi el 100\% de los usuarios de televisión por suscripción para marzo 2017. (Zapata, 2017)

Claro es líder del mercado con una participación del $48 \%$ seguido de UNE y ha mostrado un crecimiento leve pero sostenido de $3 \%$ en los últimos 4 años.

Hasta el momento Claro es el único operador de Televisión que se está preparando para la creciente entrada de la televisión bajo plataformas digitales; para esto creo la plataforma 
"Claro Video", la cual se ha ido posicionando bajo el formato de streaming y se vislumbra como la principal competencia a mediano plazo de los grandes jugadores de este segmento.

\begin{tabular}{|l|r|r|r|r|}
\hline \multicolumn{5}{|c|}{ TABLA 1. ANALISIS MERCADO TELEVISION POR SUSCRIPCION } \\
\hline & CLARO & MOVISTAR & \multicolumn{1}{|c|}{ ETB } & \multicolumn{1}{c|}{ Tigo Une } \\
\hline USUARIOS & $2,380,126$ & 548,314 & 981,282 & $1,040,800$ \\
\hline VARIACION & $2.35 \%$ & $10.50 \%$ & $-2.25 \%$ & $4.80 \%$ \\
\hline PARTICIPACION MERCADO & $\mathbf{4 8 \%}$ & $11 \%$ & $20 \%$ & $21 \%$ \\
\hline
\end{tabular}

Fuente: MINTIC DIC 2016

Elaboración propia (2019)

En el segmento de telefonía fija; aunque Claro permanece como Líder desde hace varios años; este es el único segmento donde no tiene una ventaja significativa sobre sus competidores. En general este segmento y acorde a la tendencia mundial ha mostrado un decrecimiento de suscriptores sostenida en el tiempo; esto debido principalmente al aumento generalizado del uso de las líneas celulares.

TABLA 2. ANALISIS MERCADO TELEFONIA FIJA HOGAR/EMPRESARIAL

\begin{tabular}{|l|r|r|r|r|r|}
\hline & CLARO & MOVISTAR & \multicolumn{1}{c|}{ ETB } & \multicolumn{1}{c|}{ Tigo Une } & TOTAL \\
\hline USUARIOS & $1,757,385$ & $1,387,638$ & $1,339,178$ & $1,407,263$ & $5,891,464$ \\
\hline VARIACION & $-1.89 \%$ & $-2.37 \%$ & $-1.30 \%$ & $0.30 \%$ & \\
\hline PARTICIPACION MERCADO & $\mathbf{3 0 \%}$ & $24 \%$ & $23 \%$ & $24 \%$ & \\
\hline
\end{tabular}

Fuente: MINTIC DIC 2016

Elaboración propia

En general Claro es el líder dominante del mercado de las telecomunicaciones en Colombia, define las tendencias del sector y ha mantenido esta misma posición dominante con estrategias de servicio al cliente, precios competitivos, aumento y mejoramiento permanente de su infraestructura, así mismo, invierte parte de sus ingresos en mejoramiento de instalaciones, investigación y desarrollo.

El mercado de Colombia es un mercado maduro; las cifras de crecimiento de todos los operadores, según el Ministerio de Tecnologías de la Información y las Comunicaciones de Colombia (Min tic, 2017), demuestran que tienen un cubrimiento cercano al $93 \%$ del mercado potencial; esto conlleva que el gran desafío de todos los jugadores del mercado de telecomunicaciones en Colombia y en especial de Claro como líder dominante es innovar, buscando nuevos mercados acorde al avance de las tecnologías, prepararse para una disminución paulatinamente significativa del mercado de telefonía fija y fortalecer sus canales de TV por suscripción generando plataformas digitales completas y que puedan competir con los grandes jugadores del mercado actual. 


\section{Importancia de Claro en el desarrollo de las telecomunicaciones en Colombia.}

El aporte de Claro en cabeza de su predecesora Comcel S.A. en el desarrollo de las telecomunicaciones en Colombia es innegable; cuando inició operaciones en el país y en su afán de alcanzar el liderato del mercado sentó las bases de la infraestructura necesaria para generar el cubrimiento actual del $99.1 \%$ de todo el territorio nacional. (Escuela Nacional Sindical, 2016)

Sus procesos de innovación en estrategias de mercadeo, como ser el pionero en el uso de la tecnología satelital para garantizar cobertura en zonas alejadas y la inserción de la telefonía móvil bajo la modalidad de prepago, produjeron un movimiento brusco en las tarifas de la telefonía móvil que en ese momento aún no eran accesibles al grueso de la población, esto conllevó a una masificación del servicio lo que redundo en menores tarifas a la población en general.
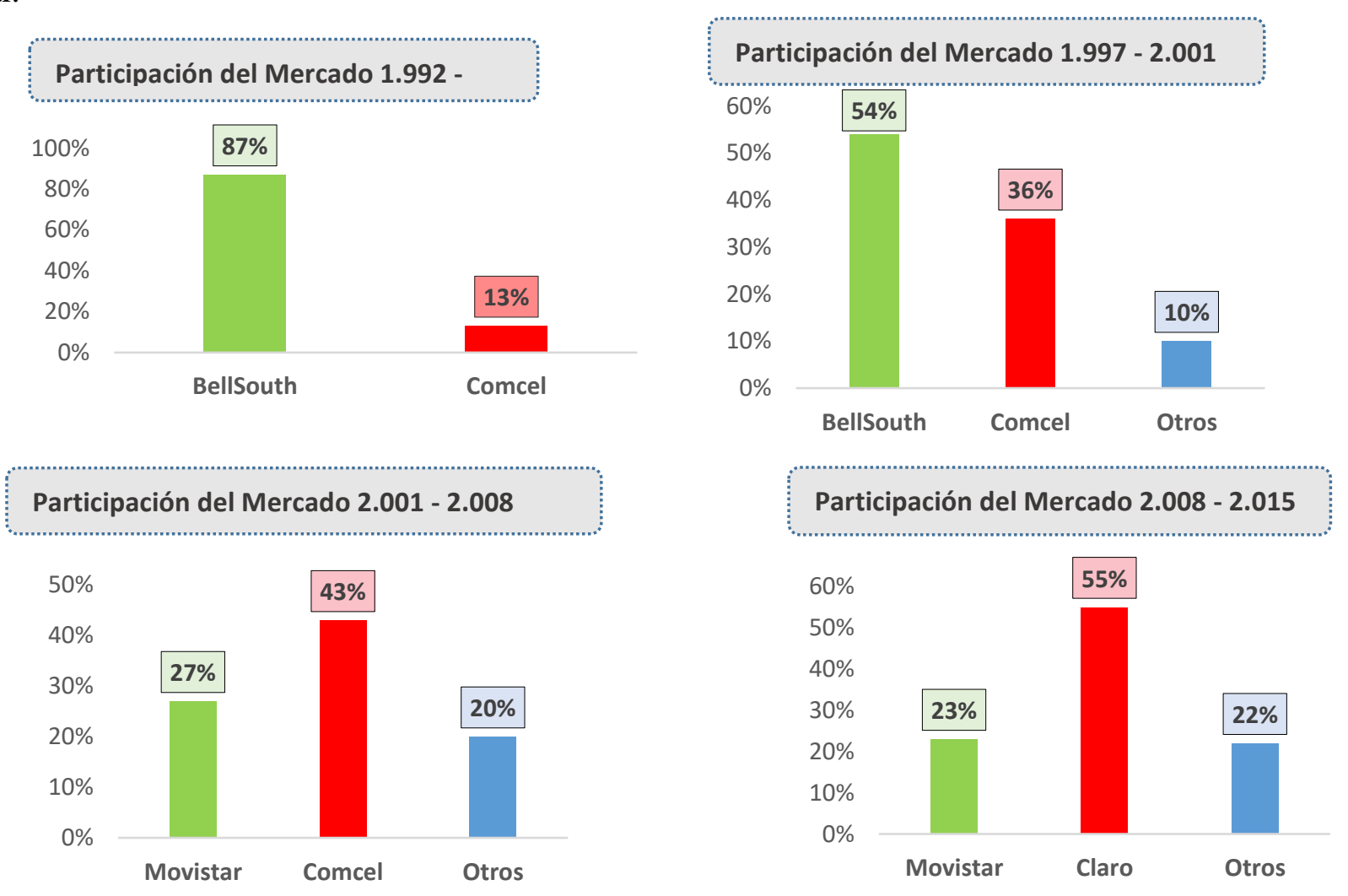

Colombia (Mintic, 2017) había en Colombia 60,73 millones de líneas celulares activas, es decir casi 1.4 por cada habitante, de estos el $73 \%$ eran líneas prepago, demostrando a simple vista el impacto que tuvo las estrategias de mercadeo de Claro en la vida de los colombianos, además de la inclusión de nuevas tendencias como "claro música" que lo mantienen a la vanguardia de los avances tecnológicos y las tendencias de consumo de la población en general. 


\section{Metodología}

La metodología del presente artículo es cualitativa con alcance descriptiva, basada en el rastreo bibliográfico; con aportes de análisis de los autores del mismo en la prospectiva del sector de las telecomunicaciones.

\section{Prospectiva del negocio de Telecomunicaciones}

El negocio de las telecomunicaciones es un grito de batalla y punto de lanza de las tecnologías de punta, debatir de cómo será o hacia donde girará el negocio de las telecomunicaciones se convierte en una rueda de la fortuna; sin embargo, diversos autores tales como Peter Foyo y Don Tapscott coinciden en diversos aspectos que mostramos a continuación:

Telefonía Fija: Indudablemente desaparecerá en el mediano plazo; haciendo una analogía será como encontrar un teléfono público en la actualidad.

Telefonía Móvil: Esta mutará tal como la conocemos ahora; habrá un cambio en la tecnología y las llamadas de voz se realizarán a través de la red, así mismo los teléfonos celulares se caracterizarán por su gran capacidad de procesamiento de aplicaciones cada vez más complejas y todo cada vez estará más conectado a estos aparatos; prácticamente toda la vida del futuro girará en torno a estos elementos. (Uso de celulares por cada 100 habitantes , s.f.) Televisión: Gradualmente tornara a aplicaciones en streaming en plataformas digitales; la consigna es "yo veo lo que escojo y no lo que me toca". (Foyo)

A nivel poblacional la juventud tecnológica actual considerará el acceso a Internet; aplicaciones de streaming y acceso a un sin número de aplicaciones como un derecho y no como un privilegio por el que haya que pagar; a futuro los autores consideran que el servicio mutará hacia algo similar a un impuesto que sería recaudado ya sea por el gobierno o por los operadores; en términos generales se espera que para 2050 todo el planeta este interconectado y todos los dispositivos y servicios estén completamente estandarizados. (Zapata, 2017)

A nivel particular de Colombia y debido a la globalización no debería haber ninguna diferencia entre las tendencias mundiales en las telecomunicaciones y las aplicaciones de la misma tecnología en el país; sin embargo, se podría inferir un pequeño retraso en la implementación después de que se ponga en funcionamiento en países más desarrollados.

Se calcula que para el año 2020; el mundo empezará la conexión 5G a gran escala; esta tecnología no significa sólo rapidez en la conectividad; este es un cambio completo de tecnología que afectara gran cantidad de dispositivos electrónicos, tales como electrodomésticos, computadores, hogares inteligentes, computadores, celulares, etc. Se calcula que el 74\% de la inversión en el sector de telecomunicaciones hasta el año 2030 se realizará en adecuación y/o cambios de redes, adaptando toda la tecnología actual al creciente uso del sistema multimodal que traerá la tecnología $5 \mathrm{G}$ y posterior. (Zapata, 2017) 


\section{Cómo se está preparando Claro hacia el Futuro.}

El sector de las telecomunicaciones, donde se desarrolla el negocio de Claro S.A, es el sector donde según estudios se produce un cambio de tecnología en el menor lapso de tiempo comparado con otro tipo de Industrias; en los últimos 20 años el sector se ha visto afectada por el cambio de 3 tecnologías tales como Internet; comunicación IP; Conexión Satelital.

Estos cambios en un lapso tan corto han generado que sólo las industrias más fuertes puedan sostenerse proveyendo servicios a una sociedad cada vez más exigente.

Uno de los mayores Retos de Claro S.A en el negocio de las comunicaciones es la competencia disruptiva; entendiéndose por esta como la aparición cada vez mayor de start's up enfocadas en la comunicación de las personas, así como la mutación de servicios complementarios que están ofreciendo otras empresas convirtiéndolas en directas competidoras de Claro S.A, como un ejemplo de esto podemos nombrar el servicio de Voz ofrecido por WhatsApp o últimamente por Facebook; los mismos que en su momento prácticamente acabaron con el negocio de los mensajes MSM, los cuales eran considerados un rubro importante de facturación dentro de los jugadores del negocio de las telecomunicaciones. Claro S.A: como Líder del Mercado es conocedor de esta situación y según datos del Ministerio de Tecnologías de la Información y las Comunicaciones de Colombia (Mintic, 2017) para Noviembre del 2017 solicito ampliación de espectro de Telecomunicaciones y aclaración del marco legal para la implementación de la Tecnología 5G en el país, además las inversiones planeadas a largo plazo 2020 - 2030 para la implementación de esta tecnología duplicarían a la mayor inversión de Claro en el país desde que según su estrategia implementada desde 2008 - 2012 inicio la red satelital e integró miles de antenas repetidoras a lo largo del país para asegurar la mayor cobertura posible en el país y que a la postre lo convirtió en el líder del Segmento. (Acosta F. , 2017)

La decisión de Claro de invertir en la mayor red 5G del país es un indicador de que se está preparando hacia el futuro; analizando la prospectiva de las telecomunicaciones; las llamadas voz a voz usando la red PIP (Punto a Punto) tenderán a desaparecer y las llamadas de voz se harán a través de la Red o bajo plataformas especiales; bajo ese esquema será de vital importancia tener una red rápida, confiable y que permita la mayor interacción de la sociedad como lo será según los analistas la nueva red 5G.

En cuanto al negocio de la Televisión por Suscripción; Claro S.A. ya ha dado muestras de estar a la vanguardia de los gustos de los consumidores y que además conoce el destino de su negocio a largo plazo; la implementación de su servicio de Streaming Claro Video, es una prueba fundamental de cómo la empresa se prepara hacia el futuro y como realiza inversiones tecnológicas para mantenerse a la vanguardia del sector. En la actualidad Claro es la única empresa de TV por suscripción que presta el servicio de streaming con su plataforma propia; a mediano plazo entrará a competir con los grandes jugadores de este segmento como Netflix y Amazon.

Para la comunicación fija se presenta el mayor reto para Claro; la tendencia en los hogares es que para el año 2035 se habrán reducido las líneas telefónicas fija en un $63 \%$ y en las 
empresas se calcula una reducción del 26\%; sin embargo en el ramo empresarial existe una alta tendencia hacia la digitalización, lo cual incluye la comunicación; con esto la empresa debe mutar a ofrecer multiservicios tales como manejo información, archivo digital, análisis de datos, marketing digital y comunicaciones. En Colombia aún no hay indicios que la empresa esté mutando a este tipo de servicios; sin embargo; en otros Países de la Región como México se han empezado desarrollos tecnológicos de este estilo; lo que se esperaría es que poco a poco empiecen a implementarse en los otros países donde el conglomerado de empresas tiene presencia en el mundo.

Claro a través del tiempo se ha caracterizado por estar en constante progreso respecto a los avances tecnológicos y en ocasiones ha marcado la tendencia en los primeros cambios generacionales de la industria de Telecomunicaciones en Colombia, por eso frente a los nuevos retos que presenta el ingreso de nuevas tecnologías ya se ha ido preparándose para estar a la vanguardia de la implementación de estos cambios en el país que le garanticen la permanencia como líder indiscutible del Mercado Colombiano.

\section{Enfoque de responsabilidad Social de Claro}

Actualmente Claro Colombia cuenta con 29.352.831 clientes de servicios de telefonía móvil, cubriendo $99.1 \%$ de municipios del país, 2.219 .618 suscriptores de internet fijo, 1.733.449 de usuarios de telefonía fija y 2.376.440 suscriptores de televisión.

Es una empresa que influye positivamente en la generación de empleo en el país. Actualmente trabajan más de 9.575 personas (8.366 Colaboradores directos y 1.209 colaboradores temporales y aprendices). Toda su fuerza de trabajo participa constantemente en programas de desarrollo y aprendizaje para fortalecer las habilidades de los líderes y motivar equipos a través de talleres de cohesión para afianzar sus competencias.

Así mismo, y con el auge del calentamiento global y las energías renovables en pro de cuidar el medio ambiente, Claro Colombia como empresa insignia de las telecomunicaciones del país, dentro de sus principales estrategias, está trabajando en implementar infraestructuras que generen consumos mínimos de energía y manejo de las emisiones de $\mathrm{CO} 2$, adicionalmente generan programas para la recolección y reutilización de equipos electrónicos, incluidos dentro de sus campañas publicitarias e imagen corporativa.

\section{Discusión}

Después de analizar la transformación de las telecomunicaciones en Colombia, y como este sector ha ido evolucionando, se evidencia que la llegada de los sistemas móviles de comunicación fue en primera medida un gran avance tecnológico para el país, adicionalmente debemos mencionar el enorme beneficio que trajo a la comunidad en general, ya que permitió que los rincones más alejados del país se conectaran.

Por otro lado, se resalta el compromiso por parte de la empresa Claro para mantenerse a la vanguardia de las nuevas tendencias tecnológicas permitiendo a los colombianos estar 
actualizados, esto reflejado en su portafolio de servicios, el cual permanece en constante renovación.

Lo anterior sumado a sus políticas de responsabilidad social y el cuidado del medio ambiente han hecho que la gestión e impacto de la empresa Claro en Colombia sea exitosa, convirtiéndola en la empresa de telecomunicaciones número uno del país.

En General Claro Colombia es reconocida por ser una de las empresas más importantes y solidas del país, no solo por la prestación de sus servicios, ayudando a que los rincones más apartados del país se encuentren comunicados con las principales ciudades, sino que es considerada como una de las empresas generadoras de empleo predilecta por los colombianos.

El desarrollo del negocio de las telecomunicaciones en Colombia ha estado direccionado por dos grandes compañías, Claro y Telefónica, sin embargo, aunque Claro no tenía una posición dominante en sus inicios; la estrategia de expansión de infraestructura, su foco en cobertura y su inclinación a buscar servicios de calidad a precios accesibles; generaron un cambio en su posicionamiento del mercado y la convirtió de lejos en la empresa más importante del sector, es en este punto cuando el análisis realizado demuestra que la expansión de Claro realmente se convirtió en la expansión de la red de comunicación de todo un país, podemos concluir que Claro conectó a un país generando desarrollo, nuevas formas de vida, de estudio y en general un mayor bienestar para la comunidad en general, bienestar que siempre viene acompañado de la innovación tecnológica. 


\section{Conclusiones}

El principal éxito de la telefonía celular en Colombia se debe a factores muy particulares, como la falta de líneas convencionales para un país con grandes necesidades de comunicación y la implantación desde un principio del esquema "la persona que llama paga" y el éxito de la telefonía prepagada representado en las tarjetas prepago, las cuales les brindaban la posibilidad a las personas de comunicarse fácilmente y a precios cómodos. Adicionalmente, los precios decrecientes, el aumento del mercado y las agresivas campañas publicitarias y promocionales le dieron un enorme crecimiento inicial a este servicio, el cual se ha mantenido gracias a los avances tecnológicos.

Claro Colombia desde sus inicios ha sentado las bases de la transformación tecnológica del negocio de telecomunicaciones en el país, se ha caracterizado por estar a la vanguardia en prestación de servicios, infraestructura de telefonía móvil y fija, convirtiéndola en un elemento accesible a toda la población colombiana, ratificándose como la principal empresa de telecomunicaciones del país por su amplia cobertura.

Sus estrategias comerciales han contribuido al desarrollo exponencial del sector colocando al país en una cobertura prácticamente total equiparable a los países desarrollados del mundo.

Adicionalmente, Claro es una empresa sostenible por su constante innovación en su portafolio de servicios, productos, facilidades de pago, implementación de plataformas digitales de fácil acceso, atención al cliente, etc. Todo en base en ir de la mano con los avances tecnológicos utilizándolos a su favor, manteniéndose a la vanguardia en el campo de la telecomunicación, por encima de sus competidores.

En General Claro S.A y sus predecesoras han sido líderes del mercado desde el año 2008 hasta la actualidad y con si visión del negocio a largo plazo; la inversión planeada en Tecnología se espera que su posición dominante en el mercado colombiano no tenga ningún cambio significativo a futuro. 


\section{Referencias}

Acosta. (2017). CTR, Analisis de los mercados moviles e internet en colombia. Obtenido de www.mintic.gov.co/portal/604/w3-article-51966.html

Acosta, F. (2017). La privatizacion de las comunicaciones en Colombia. Bogota: CESA.

Benavides, J., \& Castro, F. (2012). Promocion de la competencia en la telefonia movil en Colombia. Bogota: Fedesarrollo.

Botero Rico, C (2016). Analisis del sector publico de las telecomunicaciones, Universidad de Medellin

Congreso de la Republica. (2009). Ley 1341de 2009. Bogota, Colombia. Tomado de https://www.mintic.gov.co/portal/604/w3-article-3707.html

Congreso de la Republica. (1989). Ley 72 de 1989. Bogota, Colombia. Tomado de https://mintic.gov.co/portal/604/w3-article-3720.html

Congreso de la Republica. (1990). Ley 1900 de 1990. Bogota, Colombia. Tomado de https://www.mintic.gov.co/portal/604/articles-3568_documento.pdf

CRT. (2002). Telecomunicaciones en Colombia. Tomo I años 1998 - 2001, el gran avance. Bogota: Ministerio de Telecomunicaciones.

Devis, I. (20014). Historia de las telecomunicaciones en Colombia, Ed Intermilenio

Don tapscott; growing up digital, 2008 Mc graw Hill

El Espectador. Uso de celulares por cada 100 habitantes . (s.f.). Obtenido de https://www.indexmundi.com/g/r.aspx?v=4010\&l=es

Escuela Nacional Sindical. (2016). Entramado de relaciones laborales con los gigantes colombianos. Bogota: Ediciones Escuela Nacional Sindical.

Gonzales, J. F., Sepulveda, A. L., \& Triviño, L. K. (Febrero de 2014). Estrategias del sector de la telefonia publica basica conmutada frente al actual desplazamiento de formas tradicionales de comunicacion en Colombia. Econografos Escuela de Economia, 57, $2-10$.

Hernandez, H (2016) Tecnologias de la informacion y la comunicación, Corporacion Universitaria de la Costa 
Hernandez, J (2015) Comunicaciones Moviles, Ed universitaria Ramón Areces

Herrera, H. (2008). Innovación e incumbent failure: Una ilustración usando la industria de las telecomunicaciones en Colombia . Documentos de Trabajo. Economia y Finanzas. Centro de Investigacion Economica y Financiera, 4-10.

Huidobro, J (2016) Tecnologia de Telecomunicaciones, Producciones Copyright

Matias. (2006). Politicas publicas y telecomunicaciones en Colombia. Revista Dialogos de saberes, 24.

Mojica, F (2012) La construcción del Futuro, conceptos y modelos de prospectiva estrategica, territorial y tecnologica, Universidad Externado de Colombia Muñoz, F (2014) Servicios Publicos Domiciliarios, telecomunicaciones e infraestrutura, Universidad Externado de Colombia.

Ortega, M (2016) Matlab aplicado a Telecomunicaciones, Alfaomega.

Pinto, R (2016) Fundamentos de sistemas de comunicaciones analogicas

Peter Foyo, "Enginner strike back; prospective of telecomunications bussines to 2030, deloitte 2017

Restrepo, J (2015) Ingenieria de Telecomunicaciones, conceptos basicos, Universidad de Medellin

Sarmiento, E. (25 de Septiembre de 2011). La extincion de Telecom.

Sarmiento, F (2012) Historia de la Televisión Colombiana, Mintic

Tapscott / Williams, 2006, "Wikinomics", Mc graw hill

Torres, H. (2007). Telecomunicaciones y Telematica, de las señales de humo a las redes de información, Escuela Colombiana de Ingenieria.

Zapata, L (2017) El ecosistema Digital y las autoridades de regulación de los sectores audiovisual y Tic, universidad Externado de Colombia. 